\title{
A visual timeline tool for tracking mood and medication perinatally in affective disorders.
}

\author{
Perry, A. ${ }^{1}$, Gordon-Smith, K. ${ }^{1}$, Jones, L $^{1}$. and Jones, I. ${ }^{2}$ \\ ${ }^{1}$ Department of Psychological Medicine, Institute of Health \& Society, University of Worcester, UK. \\ ${ }^{2}$ Institute of Psychological Medicine \& Clinical Neurosciences, Cardiff University, UK.
}

\section{Corresponding author:}

\author{
Amy Perry \\ Department of Psychological Medicine \\ Institute of Health and Society \\ Breedon Building \\ St Johns Campus, \\ Henwick Grove, \\ Worcester, \\ WR2 6AJ
}

Tel: 01905542803

Email: a.perry@worc.ac.uk

\section{Acknowledgements:}

The authors would like to thank the Stanley Medical Research Institute for funding this research and all members of the Bipolar Disorder Research Network (www.bdrn.org). We also wish to express our gratitude to all the individuals who kindly gave their time to participate in this research.

\section{Disclosure of potential conflicts of interest:}

Funding: This study is funded by the Stanley Medical Research Institute (grant number 5710002223)

Conflicts of interest: All authors report no conflicts of interest. 
Women with bipolar disorder are at particularly high risk of relapse following childbirth with almost one in five experiencing an episode of postpartum psychosis following delivery (Di Florio et al. 2013; Wesseloo et al. 2016). Factors which increase risk for severe postpartum relapse in women with bipolar disorder are poorly understood. The majority of previous research has traditionally employed retrospective methods of assessment, and/or has only measured a limited range of potential risk factors. High quality studies investigating risk factors prospectively in pregnant women with bipolar disorder are rare, and have predominantly focused on the role of medication use in postnatal relapse (Viguera et al. 2007).

We are currently conducting a large-scale prospective pregnancy study to investigate factors which influence risk for severe postpartum mood episodes in women with bipolar disorder (UK Bipolar Disorder Research Network, www.BDRN.org). Women are recruited systematically through specialist perinatal psychiatry services nationwide, or non-systematically via national patient support charities. Lifetime and current psychopathology is assessed during pregnancy using a semi-structured interview (Schedules for Clinical Assessment in Neuropsychiatry; Wing et al. 1990), and a follow-up SCAN interview is conducted at 12-weeks postpartum to assess psychopathology during the perinatal period. These data are combined with further information obtained at 12-weeks postpartum from clinician questionnaires and medical case records to determine both the occurrence of episodes of illness and medication use across the whole perinatal period. Data are also gathered on a wide range of potential risk and protective factors including obstetric complications, sleep loss, psychosocial factors relating to pregnancy (social support for example), comorbid physical and psychiatric conditions, menstrual history and major adverse life events occurring lifetime ever and in the perinatal period specifically.

These data are highly complex and multifaceted; there is considerable variation not only between participants but also individually throughout the perinatal period. For research, but also in clinical practice, there is a need to summarise this complex information and we have therefore developed a visual timeline tool as a means of graphically representing these detailed psychiatric and medication data longitudinally and simply across the perinatal period. Fig. I provides an example timeline generated from data collected during the second pregnancy of a participant with DSM-5 (American Psychiatric Association 2013) bipolar I disorder. This woman, who had a previous episode of postpartum psychosis, took Quetiapine prophylactically throughout her pregnancy. She experienced an episode of major depression at 24 weeks gestation. Following an increase in the dose of Quetiapine she recovered and remained euthymic throughout the rest of the pregnancy. The Quetiapine dose was increased again at 32 weeks of pregnancy and she delivered at 37 weeks. At 24 days postpartum she developed an episode of postpartum psychosis requiring compulsory admission to a psychiatric mother and baby unit and the addition of further psychotropic medication.

We have found this timeline tool to be a simple and efficient method of collating and presenting large amounts of complex clinical data for each perinatal period. Presenting these data graphically has allowed us to visually identify potential patterns in the data that are taken forward for formal testing. In addition to its use in research, we anticipate this tool would be equally useful in clinical practice for reviewing a patient's perinatal psychiatric history.

American Psychiatric Association (2013) Diagnostic and Statistical Manual of Mental Disorders, 5th edn. American Psychiatric Publishing, Arlington, VA

Di Florio A, Forty L, Gordon-Smith K, et al (2013) Perinatal episodes across the mood disorder spectrum. JAMA psychiatry 70:168-75.

Viguera AC, Whitfield T, Baldessarini RJ, et al (2007) Risk of recurrence in women with bipolar disorder during pregnancy: prospective study of mood stabilizer discontinuation. The American journal of psychiatry 164:1817-24; quiz 1923. doi: 10.1176/appi.ajp.2007.06101639

Wesseloo R, Kamperman AM, Munk-Olsen T, et al (2016) Risk of Postpartum Relapse in Bipolar Disorder and Postpartum Psychosis: A Systematic Review and Meta-Analysis. American Journal of Psychiatry 173:117-127. doi: 10.1176/appi.ajp.2015.15010124

Wing JK, Babor T, Brugha T, et al (1990) SCAN: Schedules for Clinical Assessment in Neuropsychiatry. Archives of General Psychiatry 47:589-593. 
Fig. I A timeline representing course of illness and medication use during the perinatal period for a participant recruited to the Bipolar Disorder Research Network (BDRN) prospective pregnancy study.

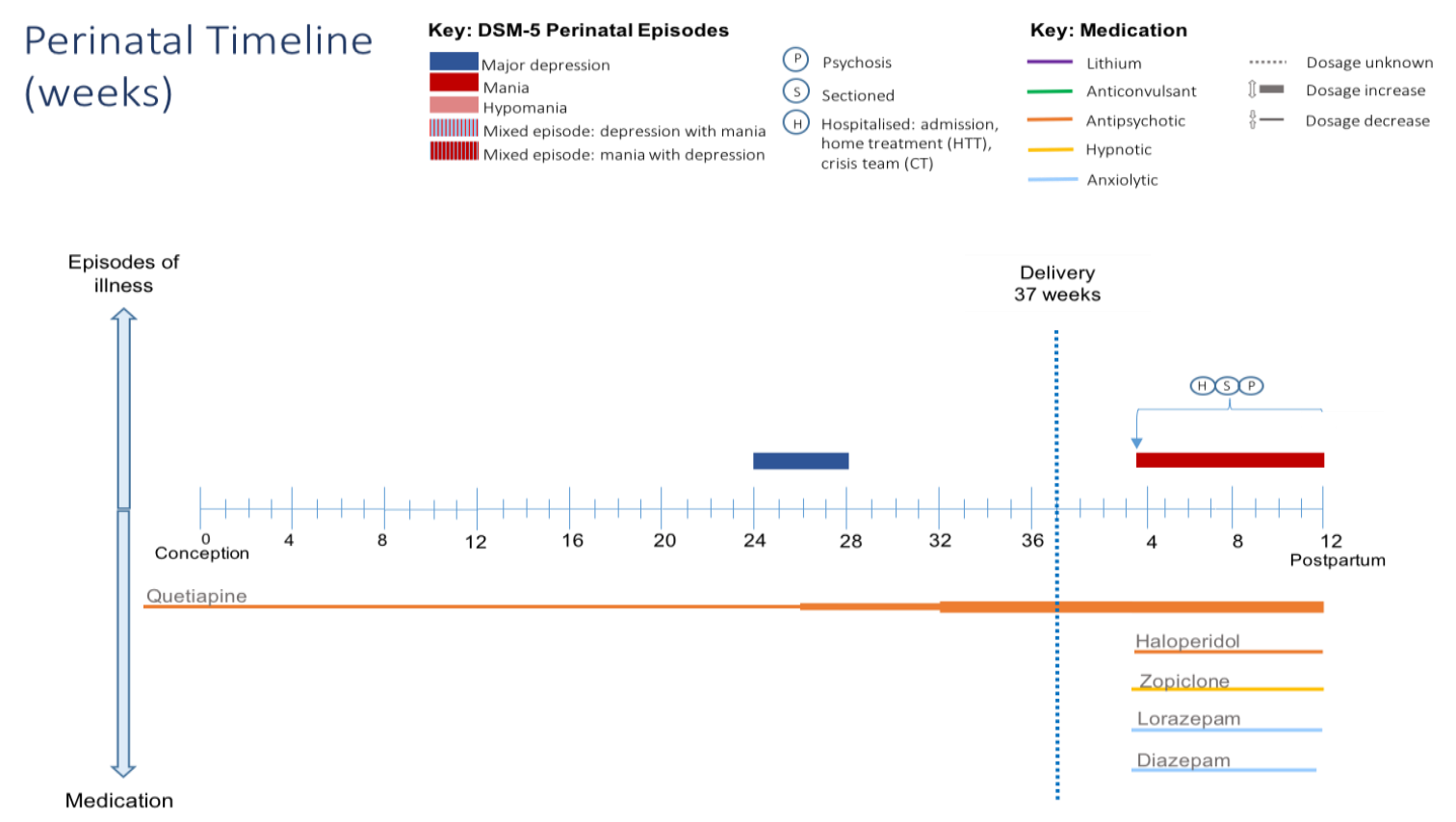

